\title{
Th17 responses in chronic allergic airway inflammation abrogate regulatory T-cell-mediated tolerance and contribute to airway remodeling
}

\author{
J Zhao $^{1}$, CM Lloyd ${ }^{2}$ and A Noble ${ }^{1}$
}

The role of T-helper type 17 (Th17) responses in airway remodeling in asthma is currently unknown. We demonstrate that both parenteral and mucosal allergen sensitization, followed by allergen inhalation, leads to Th17-biased lung immune responses. Unlike Th17 cells generated in vitro, lung Th17 cells did not produce tumor necrosis factor- $\alpha$ or interleukin (IL)-22. Eosinophilia predominated in acute inflammation, while neutrophilia and IL-17 increased in chronic disease. Allergen-induced tolerance involved Foxp3-, Helios-, and glycoprotein-A repetitions predominant-expressing regulatory T cells (Treg) and IL-10/interferon- $\gamma$ priming. This Treg phenotype was altered in inflamed lungs and abrogated by inhalation of IL-17. Using Th17-deficient mice with genetic disruption of gp130 in T cells, we showed that Th17 cells induce airway remodeling independent of the Th2 response. All-trans retinoic acid administration ameliorated Th17mediated disease and increased Treg activity, while dexamethasone inhibited eosinophilia but not neutrophilia, and enhanced Th17 development in vitro. Targeting the Th17/Treg axis might therefore be therapeutic in neutrophilic and glucocorticoid-refractory asthma.

\section{INTRODUCTION}

The key pathological features of asthma are airway hyper-reactivity and remodeling, both of which are generally attributed to activities of allergen-driven T-helper type 2 (Th2) cells and associated allergic inflammation. On the basis of animal models of acute and chronic allergen exposure, airway remodeling has been mainly associated with chronic lung inflammation, while hyperreactivity is most prominent during acute phases of the disease. This suggests prolonged exposure of lung tissue to products of Th2 cells (e.g., interleukin (IL)-13, ${ }^{1}$ eosinophils, ${ }^{2}$ or transforming growth factor (TGF) $-\beta^{3}$ leads to gradual remodeling of the tissue and decreased baseline lung function). However, recent studies in infants and young children have shown that remodeling is apparent at the earliest stages of asthma development, suggesting that both features develop in parallel. ${ }^{4}$

Asthmatic inflammation is primarily driven by allergenspecific CD4 T cells, which recruit a complex network of innate immune cells into the airways. CD4 T cells can differentiate along four distinct pathways after antigen exposure, directed by T-bet (Th1), GATA-3 (Th2), ROR $\gamma \mathrm{t}$ (Th17), or
Foxp3 (regulatory T cells (Treg)) transcription factors. Th17 cytokines have been implicated in the immunopathology of both asthma and chronic obstructive pulmonary disease. ${ }^{5,6}$ IL-17 (i.e., IL-17A) is a pro-inflammatory cytokine and IL-17deficient mice develop reduced acute inflammatory responses in a classic mouse asthma model. ${ }^{7}$ Th17 effectors recruit neutrophils into the airway as the IL-17 they produce induces secretion of IL-8, an important neutrophil-recruiting chemokine, from airway epithelial cells ${ }^{8}$ and smooth muscle. ${ }^{9}$ Moreover, IL-17 stimulates release of IL-6 from human bronchial fibroblasts and granulocyte-colony-stimulating factor expression in bronchial epithelial cells, which stimulates neutrophil development and granulopoiesis. ${ }^{10}$

Adoptive transfer of in vitro-derived Th17 cells into allergenchallenged mice induces airway hyper-reactivity and glucocorticoid-resistant inflammation. ${ }^{11}$ On the other hand, IL-17 produced by $\gamma \delta \mathrm{T}$ cells promotes resolution of pre-established allergic airway inflammation, indicating distinct roles for IL-17 during sensitization and challenge phases. ${ }^{12}$ Furthermore, skewing Th2 inflammation towards Th17 in an acute asthma

\footnotetext{
${ }^{1}$ Department of Asthma Allergy and Respiratory Science, MRC and Asthma UK Centre in Allergic Mechanisms of Asthma, King's College London, London, UK. ${ }^{2} \mathrm{MRC}$ and Asthma UK Centre in Allergic Mechanisms of Asthma, National Heart and Lung Institute, Imperial College London, London, UK. Correspondence: A Noble (alistair.noble@kcl.ac.uk) 
model inhibited airway hyper-reactivity. ${ }^{13}$ However, the role of Th17 cells in chronic asthmatic disease and airway remodeling is not known.

Airways harbor many microbes and allergens that do not result in persistent inflammation, and this tolerance is partly mediated by Treg. Th17 cells are closely related to the Foxp $3^{+}$ Treg lineage. Although the majority of Foxp $3^{+}$Treg are thymically generated, Foxp3 Treg can be peripherally induced by TGF- $\beta$ and IL-2. Conversely, TGF- $\beta$ in the presence of IL-6 directs Th17 development. Expression of transcription factor Helios has been proposed as a marker for natural, thymic Treg, with low Helios expression in induced Treg. ${ }^{14}$ As the balance between Treg and Th17 responses may be controlled by IL-6, the IL-6 pathway represents a potential therapeutic target in inflammatory disease. ${ }^{15}$ Here, we characterized Th17 responses in acute and chronic airway inflammation triggered by parenteral or mucosal sensitization and suggest that naturally induced lung Th17 cells have a different phenotype from in vitro polarized cells. We tested experimental disruption of the Th17 response using alltrans retinoic acid (ATRA) treatment or genetic deletion of IL-6R signaling in T cells. Our data establish an important role for Th17 cells in airway remodeling and chronic neutrophilia, independent of allergic inflammation. Targeting the Th17 pathway in neutrophilic asthma is therefore of potential therapeutic benefit.

\section{RESULTS \\ Primary T-cell responses to inhaled allergen induce a mixed Th17/Th2/Treg phenotype}

We first examined primary T-cell responses to inhaled allergen to characterize responses primed via the lung mucosa. This avoided effects of artificial adjuvants on the response. T-cell receptor-transgenic OT-2 CD4 cells (ovalbumin (OVA)-specific) were labeled with carboxyfluorescein succinimidyl ester (CFSE) and transferred into naïve recipients given OVA allergen alone intranasally (Figure 1a). Within 3 days, OT-2 cells were present in mediastinal lymph node (LN) but not lung tissue and had undergone extensive clonal expansion, as demonstrated by CFSE dilution. After 4-6 divisions, some cells had developed into Th17 $\left(\mathrm{IL}-17^{+}\right)$and Th2 $\left(\mathrm{IL}-4^{+}\right)$effectors, but none were Th1-like. This contrasts with in vitro characteristics of OT-2 cells, which have a strong Th1 bias (see Figure 7a). As allergen alone given intranasally is known to induce tolerance, we performed the same analysis with the addition of the mucosal adjuvant tumor necrosis factor- $\alpha$ (TNF- $\alpha$ ) to the allergen, which is known to induce allergic sensitization. ${ }^{16}$ Primary responses were enhanced (Figure 1b) in terms of both numbers of divisions and proportions of cytokine-producing cells. We also determined whether Treg cells present in OT-2 mice responded to intranasal allergen priming. Foxp $3^{+}$cells expanded to the same extent as Foxp $3^{-}$CD4 cells, and their division was also enhanced by the mucosal adjuvant (Figure 1b).

\section{Acute allergic airway inflammation is dominated by Th17 cells with a phenotype distinct from in vitro-generated Th17}

The Th17-priming environment of the lung was confirmed in B6 mice sensitized with OVA/alum and challenged acutely with OVA, the protocol most frequently used as an experimental asthma model (Figure 1c-g). T cells infiltrating the airways contained twice as many $\mathrm{IL}-17^{+} \mathrm{CD} 4$ cells as IL- $4^{+}$Th 2 cells (Figure 1c), and this Th17 predominance was more pronounced in lung CD4 cells (Figure 1d). OVA/alumsensitized animals showed no increase in CD4 cells producing Th1 cytokines or IL-22. Surface expression of the Th17 marker CCR6 and intracellular staining for the Th17 transcription factor ROR $\gamma \mathrm{t}$ were both increased on lung CD4 T cells (Figure 1e). Furthermore, significant increases in IL-17A, as well as the Th2 cytokine IL-13, were detected in bronchoalveolar lavage (BAL) fluids from mice with acute allergic inflammation, and the Th17-promoting cytokine IL-23 was also increased (Figure 1f). Stimulation of lung cells with OVA + anti-CD28 in vitro also induced IL-17A production in lung CD4 cells, suggesting that the Th17 cells were allergen specific or at least dependent on allergen priming (Figure 1g). Lung Th17 cells clearly did not stain for TNF- $\alpha$, unlike in vitro-generated IL- $17^{+}$cells (Figure $1 \mathbf{h}$ ), which were nearly all TNF- $\alpha^{+}$despite having downregulated interferon (IFN) $-\gamma$ production under Th17-polarizing conditions. There was also a difference, although not as dramatic, in IL-22 production-a substantial fraction of in vitro Th17 cells produced IL-22, while very few IL-22+ cells were found in the lung. CCR6 expression did not correlate with Th17 development in vitro (not shown).

\section{Chronic allergic airway inflammation is dominated by Th17 responses and neutrophilia irrespective of the type of sensitization or route of exposure}

Mice were sensitized with OVA combined with either the classic Th2-skewing adjuvant alum, Th17-promoting complete Freund's adjuvant (CFA) ${ }^{17}$ or Th1-skewing CASAC (combined adjuvant for synergistic activation of cellular immunity), ${ }^{18}$ and then examined for lung inflammation induced by short-term or chronic mucosal allergen exposure. Surprisingly, Th17 was the predominant phenotype with all sensitization protocols at the acute stage (Figure 2a). Both alum and complete Freund's adjuvant sensitization induced mixed eosinophilia/neutrophilia. CASAC induced only neutrophilia, with no Th1 cells apparent at the acute stage. After chronic challenge, Th17 cells and neutrophilia persisted, while fewer eosinophils were present. Irrespective of the mode of sensitization, we observed an increase in Th1 cells at the chronic stage. Thus, the route and chronicity of allergen challenge were more important than the nature of initial sensitization in directing the response. However, to ensure that our results were not influenced by systemic sensitization protocols, we compared OVA/alum sensitization with mucosal sensitization via intranasal OVA + TNF- $\alpha$ treatment (Figure 2b-d). Mucosally sensitized mice also developed a Th17-dominated response with mixed eosinophilia/ neutrophilia. However, eosinophilia was less pronounced and neutrophilia increased progressively as inflammation progressed through subchronic and chronic phases (Figure 2b). Proportions of $\mathrm{IL}-17^{+}$cells decreased at the chronic stage 
a

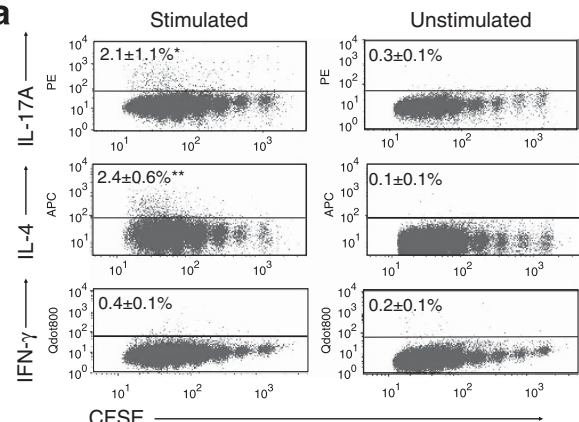

b OVA alone i.n.
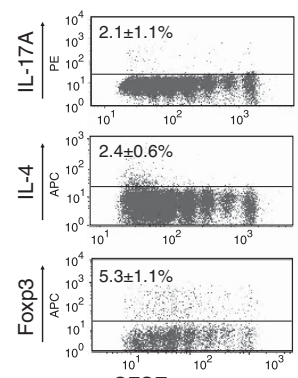

CFSE

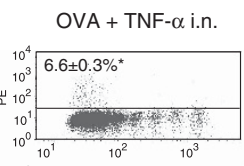

$10^{3} 3.9 \pm 0.8 \%$

: $10^{2}$
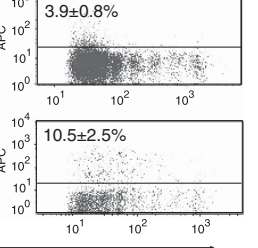

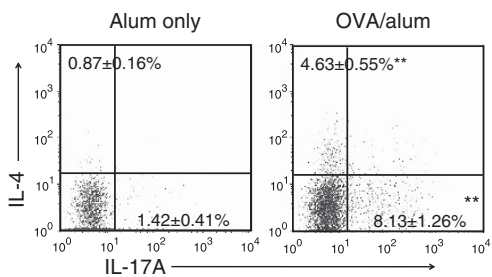

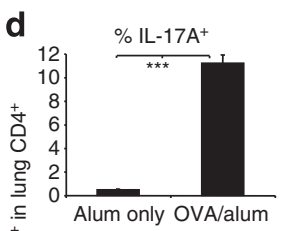
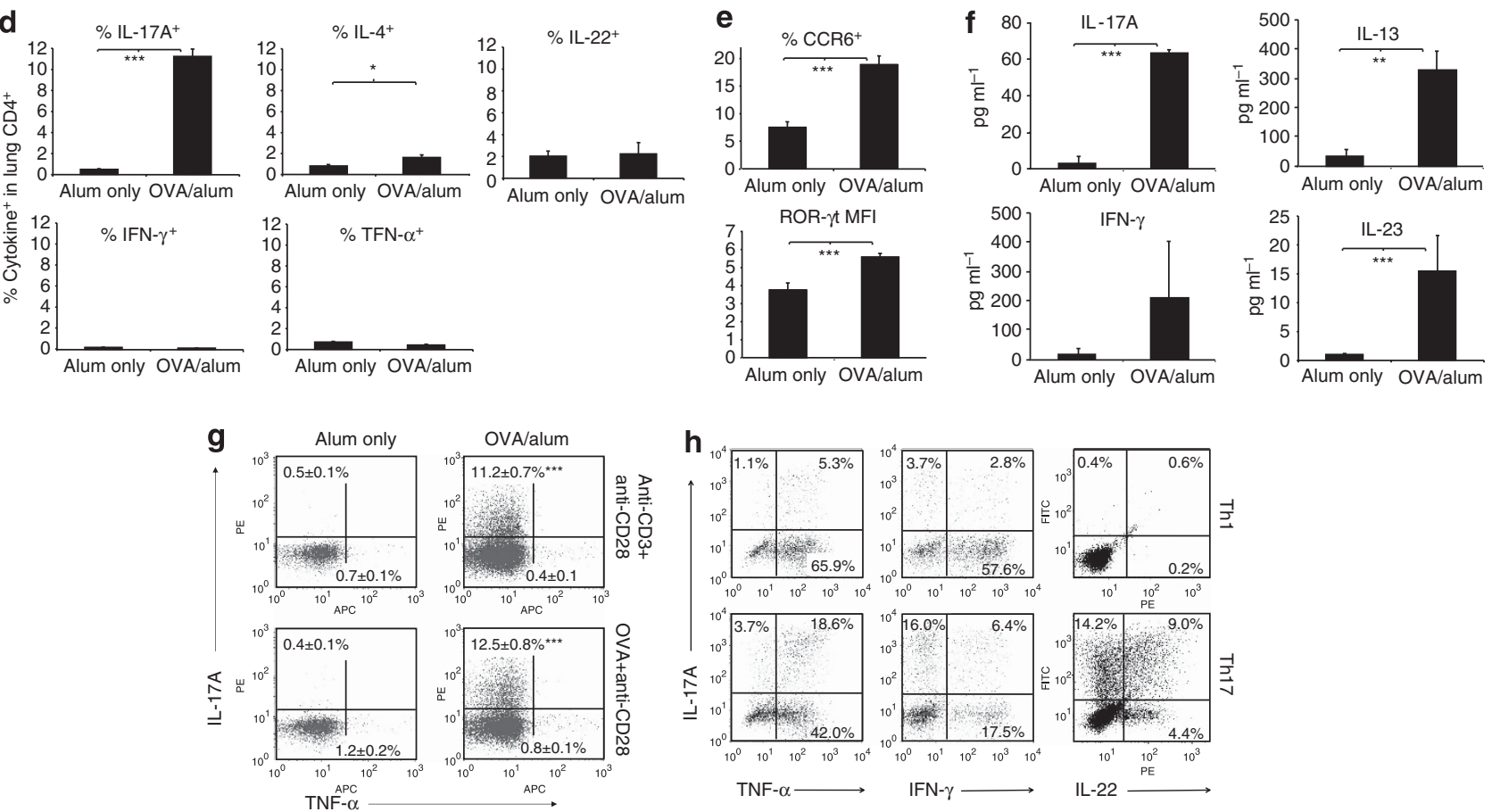

Figure 1 Acute allergic airway inflammation consists of mixed T helper (Th)17/Th2 responses. (a and b) Carboxyfluorescein succinimidyl ester (CFSE)-labeled OT-2 CD4 cells were transferred into B6 mice and primary responses to inhaled (a) ovalbumin (OVA) or (b) OVA \pm tumor necrosis factor- $\alpha$ (TNF- $\alpha$ ) determined by flow cytometry of mediastinal lymph node (LN) after 3 days. Cells were stimulated with phorbol 12-myristate 13-acetate (PMA) + ionomycin before cytokine staining or stained for Foxp3. Data are from $n=6$ mice pooled from three experiments, and statistical comparisons are to (a) unstimulated cells or (b) OVA alone. (c and d) Acute allergic airway inflammation was induced via OVA/alum sensitization or alum-alone control and OVA intranasal (i.n.) challenge. (c) Bronchoalveolar lavage (BAL) T cells or (d) lung T cells were restimulated with anti-CD3/CD28 and cytokine staining in CD4 cells is shown ( $n=5$, similar data were obtained in three independent experiments). (e) Surface CCR6 and intracellular ROR $\gamma \mathrm{t}$ staining in CD4 cells as in $\mathbf{d}$. (f) Secreted cytokines in BAL supernatants from mice as in $\mathbf{c}$. (g and $\mathbf{h})$ Lung Th17 cells are OVA-responsive and distinct in phenotype from in vitro-generated Th17: panel g shows interleukin (IL)-17 vs. TNF- $\alpha$ staining in lung CD4 cells (as in d) stimulated with anti-CD3 or OVA + anti-CD28. Panel h shows Th1 (top) and Th17 (bottom) cells generated in vitro from OT-2 CD4 cells and restimulated with PMA/ionomycin before IL-17, TNF- $\alpha$, and interferon (IFN)- $\gamma$ staining. Data are representative of three experiments. ${ }^{\star} P<0.05 ;{ }^{* \star} P<0.01 ;{ }^{* \star \star} P<0.005$.

with OVA/alum and increased with OVA/TNF- $\alpha$ sensitization (Figure 2c). IL-17A in BAL increased substantially at the chronic stage (Figure 2d) in mucosally but not parenterally sensitized mice. OVA/TNF- $\alpha$ resulted in significantly higher IL-17A and IFN- $\gamma$, but lower IL-13 levels (Figure 2d) compared with OVA/alum. Airway remodeling was observed in both models, as indicated by peribronchiolar collagen deposition and increased airway smooth muscle (shown in Supplementary Figure S1 online), and increased total lung collagen content (Figure 2e). Hematoxylin and eosin staining (Supplementary Figure S1 online) revealed pronounced leukocytic inflammation around the airways at the chronic stage in both models.

\section{Mucosal inflammation and tolerance to OVA both involve Treg} recruitment, but Treg from inflamed lungs have low Helios expression and proliferative responses

As Th17 are closely related to the Foxp 3 Treg lineage and ROR $\gamma \mathrm{t}$ and Foxp 3 are reciprocally regulated, we investigated Treg responses in mice developing tolerance or acute lung inflammation to OVA challenge. It is well known that intranasal OVA challenge of unsensitized mice leads to tolerance. 

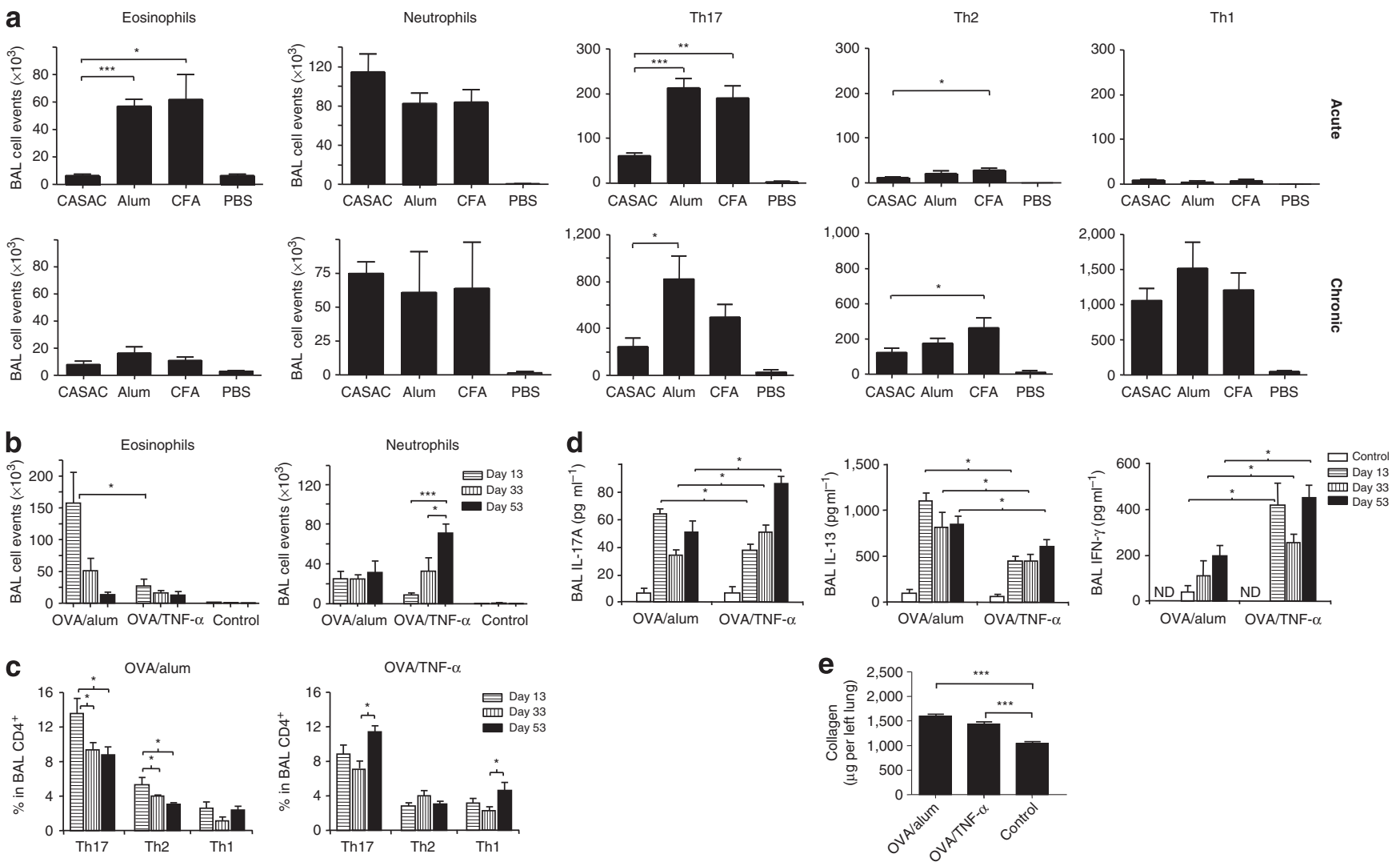

Figure 2 Thelper (Th)17 responses persist during airway remodeling induced via parenteral or mucosal sensitization. (a) Numbers of eosinophils, neutrophils, and interleukin (IL)-17 ${ }^{+}$(Th17), IL-4+ (Th2), and interferon (IFN)- $\gamma^{+}$(Th1) CD4 T cells in the bronchoalveolar lavage (BAL) of mice after parenteral sensitization with Th1- (CASAC (combined adjuvant for synergistic activation of cellular immunity)), Th2- (alum), or Th17-skewing (complete Freund's adjuvant (CFA)) adjuvants. Acute (day 13) and chronic (day 53) stages of the response (as described in the Methods section) are shown. $N=5$, similar results were obtained in two experiments. (b-d) Mucosal sensitization with ovalbumin (OVA) + tumor necrosis factor- $\alpha$ (TNF- $\alpha$ ) induces an increasingly neutrophilic and IL-17-dominated response compared with parenteral OVA/alum sensitization. (b) Eosinophilia and neutrophilia at acute (day 13) subchronic (day 33) and chronic stages of disease, and (c) \% Th1/2/17 cells in BAL CD4 cells. (d) Levels of cytokines in BAL supernatants. (e) Total lung collagen assay. $N=5$, representative of two experiments. Histological analysis of airway remodeling is shown in Supplementary Figure $\mathbf{S} 1$ online. ND, not determined; PBS, phosphate-buffered saline. ${ }^{*} P<0.05 ;{ }^{* \star} P<0.01 ;{ }^{* *} P<0.005$.

However, compared with phosphate-buffered saline (PBS) challenge, OVA challenge of mice primed only with alum induced low-level T-cell infiltration of the airways (Figure 3a). Unlike in sensitized animals, there were no eosinophils or neutrophils recruited (Figure 3a). Suspecting that infiltrating $\mathrm{T}$ cells in tolerant mice were Treg, we found increased proportions of Foxp $3^{+}$CD4 T cells in the lung but not draining LN of both tolerant and sensitized animals (Figure 3b). Expression of GARP (glycoprotein-A repetitions predominant), the Treg-associated activation marker, ${ }^{19}$ was increased in both lung and LN (Figure 3c).

To determine whether the Treg were active in response to allergen, we CFSE-labeled lung and LN cells and cultured them in OVA for 4 days before CD4/Foxp3 staining. Cells from PBSchallenged mice failed to grow and Foxp $3^{+}$cells did not survive (Figure 3d, upper panels). Cells from OVA-challenged unsensitized mice proliferated and a much higher proportion expressed Foxp3 after culture (Figure 3d-f). Interestingly, cells from inflamed lungs of sensitized mice contained high proportions of Foxp ${ }^{+}$CD4 cells, but these Treg had not divided during culture in the presence of allergen. By contrast, Foxp3CD4 cells from inflamed lung and both Treg and non-Treg from LN of either group divided extensively (Figure 3d-f). As this suggested an impairment in Treg activity in the inflammatory environment of the lung, we also determined Helios expression in the same Treg before and after culture (Figure $3 \mathbf{g}$ and $\mathbf{h}$ ). Helios expression within the Foxp $3^{+}$population was generally lower in lung cells than LN, but was increased during tolerance induction. By contrast, CD4 Treg from inflamed lung, but not LN, showed low Helios expression. The difference in Helios was most pronounced after culture in allergen (Figure $\mathbf{3 h}$ ).

\section{Respiratory tolerance involves priming for IL-10 and IFN- $\gamma$ production in CD4 $\mathrm{T}$ cells}

We measured the expression of IL-10 by intracellular staining of CD4 cells from OVA-challenged tolerant or sensitized animals. IL-10 staining was not detectable directly ex vivo or in lung cells, but could be revealed after expansion of the draining LN cells in OVA for 4 days (Figure 3i). There was little growth 

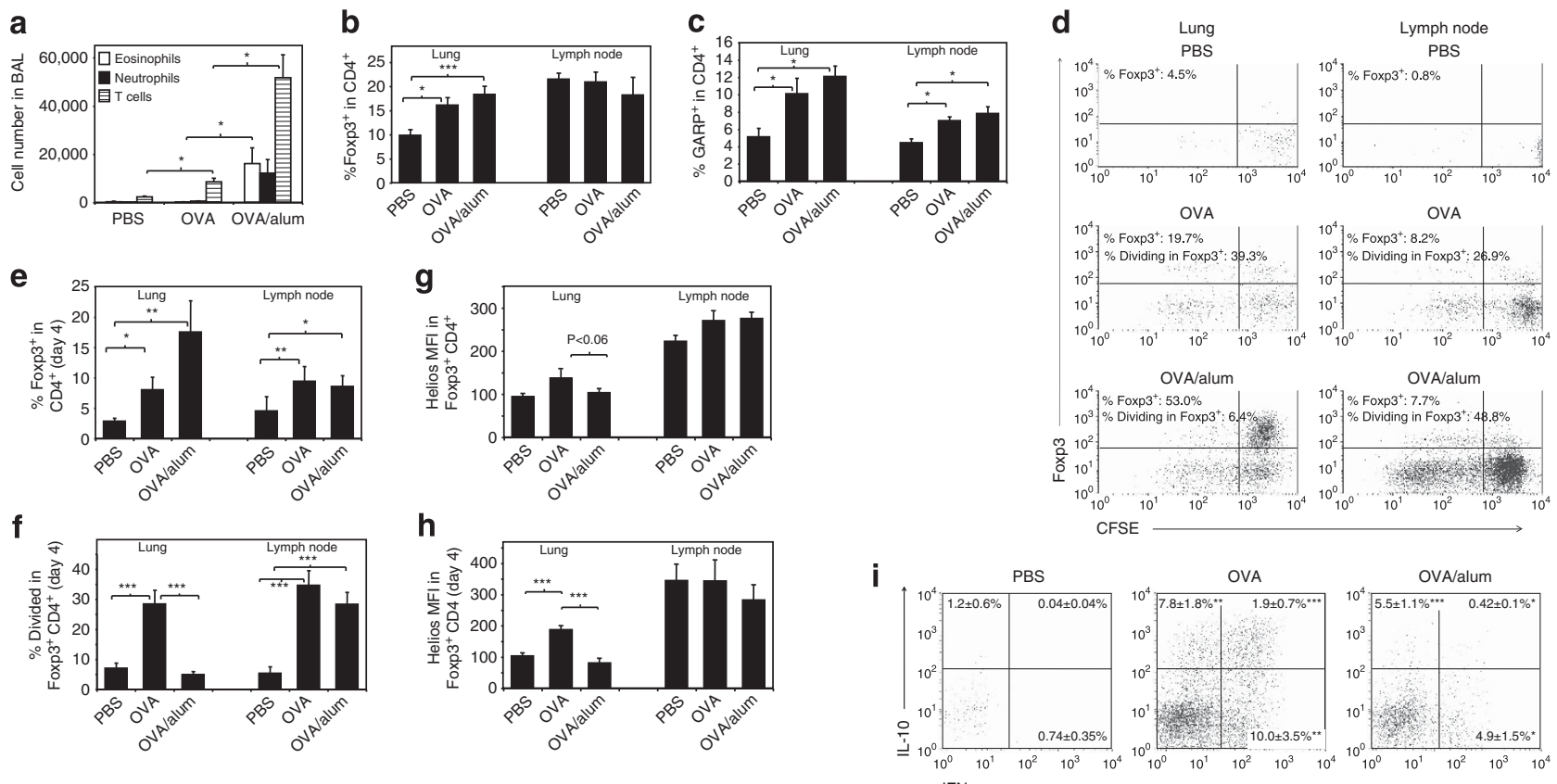

Figure 3 Respiratory tolerance involves lung regulatory T-cell (Treg) recruitment, but Treg in inflamed lung tissue are unresponsive. (a) Bronchoalveolar lavage (BAL) numbers of inflammatory cells induced by four intranasal (i.n.) doses of ovalbumin (OVA) to unsensitized mice primed with alum only (OVA, i.e., tolerance) or parenterally sensitized mice primed with OVA/alum (OVA/alum, i.e., inflammation). Naïve controls received alum alone and then phosphate-buffered saline (PBS) i.n. (PBS). $N=5$, representative of three experiments. (b) \%Foxp3 ${ }^{+}$Treg in CD4 cells of lung tissue and lymph node (LN) from mice as in a. (c) Surface glycoprotein-A repetitions predominant (GARP) expression on CD4 cells as in $\mathbf{b}$. (d-f) Cells as in b and $\mathbf{c}$ were carboxyfluorescein succinimidyl ester (CFSE)-labeled and cultured for 4 days in OVA before Foxp3 staining. Proliferation of Foxp3 ${ }^{+}$CD 4 cells was calculated as \% divided within the Foxp3 ${ }^{+} \mathrm{CD}^{+}$population. (d) Representative flow cytometry profile. (e) Pooled \%Foxp3 ${ }^{+}$data from five experiments after 4 days culture, $n=14$. (f) Pooled $\%$ divided data within Foxp3 ${ }^{+}$CD4 ${ }^{+}$populations from five experiments. (g and $\left.\mathbf{h}\right)$ Helios expression expressed as mean fluorescent intensity (MFI) of staining within Foxp3 ${ }^{+}$CD4 cells from mice as in $\mathbf{b}$, obtained (g) directly ex vivo or (h) after 4 days expansion in OVA. (i) Interleukin (IL)-10 and interferon (IFN)- $\gamma$ staining in mediastinal LN as in d cultured for 4 days in OVA and restimulated with phorbol 12-myristate 13-acetate (PMA)+ionomycin. CD4-gated events are shown, and also mean \pm s.e.m. from 12 mice pooled from four experiments. ${ }^{\star} P<0.05 ;{ }^{* \star} P<0.01 ;{ }^{* \star} P<0.005$.

or IL-10 staining in control LN cultures, but CD4 cells from tolerant mice produced IL-10 and IFN- $\gamma$, with a high proportion staining positive for both. These cytokines were reduced when animals were pre-sensitized. IL-10 could not be co-stained with Foxp3.

\section{IL-17 abrogates respiratory tolerance and enhances epithelial permeability of airways to allergen}

We asked whether IL-17 induced by allergen exposure could regulate activity of lung Treg and promote sensitization. Mice were treated intranasally with OVA alone, OVA + IL-17A, or OVA + TNF- $\alpha$ as a positive control for sensitization. After re-challenge with OVA alone, it was clear that IL-17 had induced recruitment of eosinophils and greatly increased T-cell infiltration (Figure 4a). This was however much less inflammation than that induced by TNF- $\alpha$. Furthermore, the proliferative response of Foxp $3^{+}$CD4 Treg (as in Figure 3f) in tolerant mice was abrogated after IL-17 as well as TNF- $\alpha$ exposure (Figure 4b). Priming for IL-10 production in draining LN CD4 cells was also significantly suppressed by IL-17 (Figure 4c). This abrogation of Treg activity could have been due to enhanced allergen uptake, as intranasal instillation of fluorescent-tagged OVA \pm IL-17 revealed that IL-17 promotes allergen uptake into lung tissue within $6 \mathrm{~h}$ (Figure $4 \mathrm{~d}$ ). Uptake was by both CD11c ${ }^{+}$ and $\mathrm{CD} 11 \mathrm{c}^{-}$cells, suggesting increased epithelial permeability after IL-17 exposure.

\section{Th17-deficient mice display less airway remodeling after chronic allergen challenge}

To determine the role of Th17 cells in airway remodeling, we obtained CD4-CreXgp130 flox/flox mice, ${ }^{20}$ designated here as gp130-/-, which have a gp130 deficiency targeted to the T-cell lineage. As gp130 is the signaling component of the IL- 6 receptor, these mice have $\mathrm{T}$ cells unable to respond to IL-6R signaling. Animals with the gp130 mutation, but not the CD4-Cre recombinase transgene, were used as controls and are referred to as wild type (WT). We confirmed that CD4 cells from gp $130^{-1-}$, but not controls, were deficient in Th17 development in vitro but not in Th1 or Th2 development (Figure 5a). We then compared groups of mice in which acute or chronic allergic airway inflammation had been induced, using the OVA/TNF- $\alpha$ mucosal sensitization model to maximize the chronic neutrophilic response. Lung eosinophilia was not significantly altered in the Th17-deficient strain at the acute or chronic stage, but neutrophilia was profoundly inhibited in Th17-deficient mice (Figure 5b). Levels of BAL T-cell infiltration were unchanged in gp $130^{-/-}$animals 

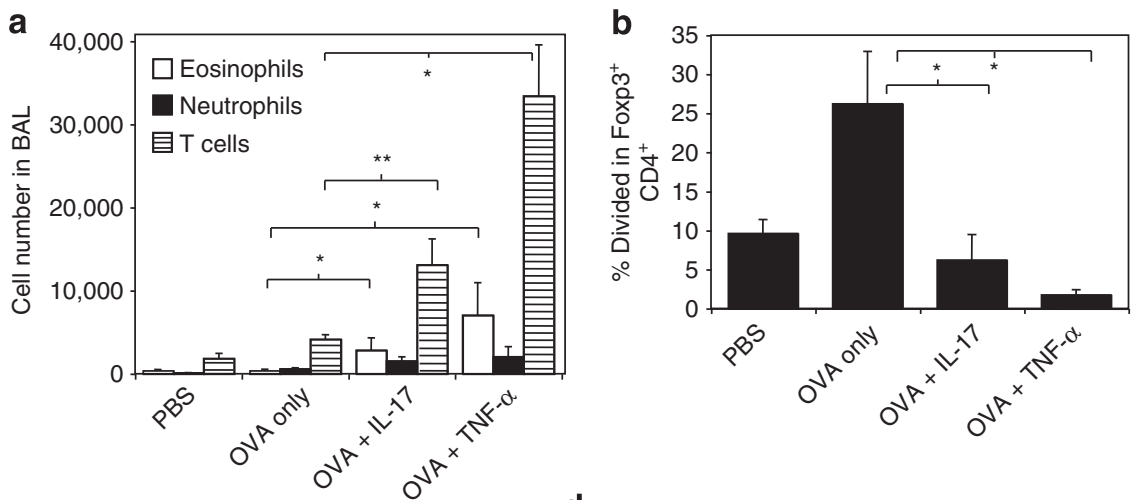

C

d

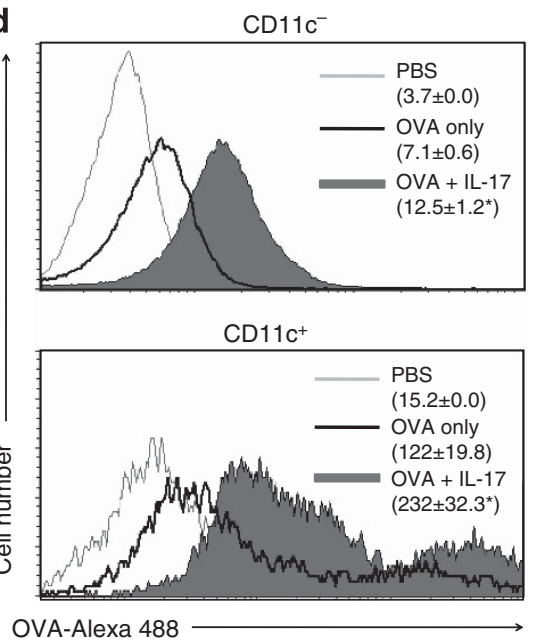

Figure 4 Interleukin (IL)-17 abrogates regulatory T-cell (Treg)-associated respiratory tolerance and enhances allergen uptake from airways to lung tissue. (a) Numbers of inflammatory cells in bronchoalveolar lavage (BAL) of mice treated intranasally (i.n.) with two doses of ovalbumin (OVA) $(50 \mu \mathrm{g}) \pm \mathrm{IL}-17(0.5 \mu \mathrm{g})$ or tumor necrosis factor- $\alpha$ (TNF- $\alpha)(1 \mu \mathrm{g})$, followed by two doses of OVA alone. Controls received phosphate-buffered saline (PBS) only. (b) Treg activity in mice as in a, assessed by carboxyfluorescein succinimidyl ester (CFSE) labeling and culture for 4 days in OVA before Foxp3 staining. Mean \% divided \pm S.e.m. from five animals is shown. (c) \% IL-10 and interferon (IFN)- $\gamma$ staining within CD4 gate from mediastinal lymph node (LN) cultured for 4 days in OVA before phorbol 12-myristate 13-acetate (PMA) + ionomycin restimulation, $n=5$. (d) Uptake of Alexa 488-labeled OVA onto lung tissue cells $6 \mathrm{~h}$ after i.n. challenge of naïve mice with $50 \mu \mathrm{g}$ OVA-Alexa $488 \pm 0.5 \mu \mathrm{g} \mathrm{IL-17}$. Histograms gated on CD11 $\mathrm{C}^{+}$dendritic cells (DCs)/macrophages and CD11 ${ }^{-}$lung cells are shown, alongside mean fluorescent intensities (MFIs) \pm s.e.m. from $n=3$ mice. Statistical comparisons to OVA-only group are shown. Results are representative of two experiments. ${ }^{\star} P<0.05 ;{ }^{* \star} P<0.01$.

at the acute stage, but were less than WT mice by the chronic stage (Figure 5c). Numbers of airway Th17 were reduced at both time points, while Th2 cells significantly increased in chronically challenged lungs from the gp $130^{-/-}$strain. The shift from a Th17 to a Th2 response in gp130-/- was confirmed by detection of lower IL-17 and higher IL-13 in BAL at both time points (Figure 5d). Although there was a trend for lower IFN- $\gamma$ in gp130 $130^{-1-}$, the Th1 response was negligible in these experiments. Most notably, at the chronic time point, the Th17-deficient animals had greatly reduced signs of airway remodeling as assessed by histology and lung collagen content (Figure 5e and f), despite the continued presence of eosinophils, Th2 cells, and IL-13 in the airways of these mice.

\section{Retinoic acid treatment alters the balance between Th17 and Treg activity}

We determined whether ATRA, the active form of vitamin A, could block Th17-associated pathology or increase Treg activity. We confirmed that ATRA specifically blocks Th17 differentiation in vitro, while not affecting Th2 or Th1 development (Figure 6a). ATRA is known to enhance TGF- $\beta$-induced Treg development in vitro. ${ }^{21}$ Here, we showed it also enhances growth/expansion of the natural Treg population (Figure 6b). $\mathrm{CD} 4{ }^{+} \mathrm{CD} 25^{+}$cell growth was enhanced by ATRA, resulting in increased yields of Foxp3-staining CD4 cells, while expansion of Foxp $3^{-}$cells in $\mathrm{CD} 4{ }^{+} \mathrm{CD} 25^{-}$cultures was inhibited. The same effect was observed using the glucocorticoid dexamethasone (DEX; Figure 6b). To determine effects of ATRA in vivo, we induced chronic allergic airway inflammation by mucosal sensitization and then commenced intraperitoneal ATRA dosing at three different time points (days 26, 35, or 45); mice were killed on day 53. Only prolonged ATRA treatment (day 26 group) significantly inhibited T-cell infiltration and neutrophilia (Figure 6c). By contrast, ATRA enhanced eosinophilia, most significantly after a shorter period of treatment (Figure 6c). Prolonged ATRA treatment significantly reduced lung collagen content (Figure 6f), but its effect on Th17 cell numbers was not statistically significant and it had no effect on Th2 cells (Figure 6d). 
a

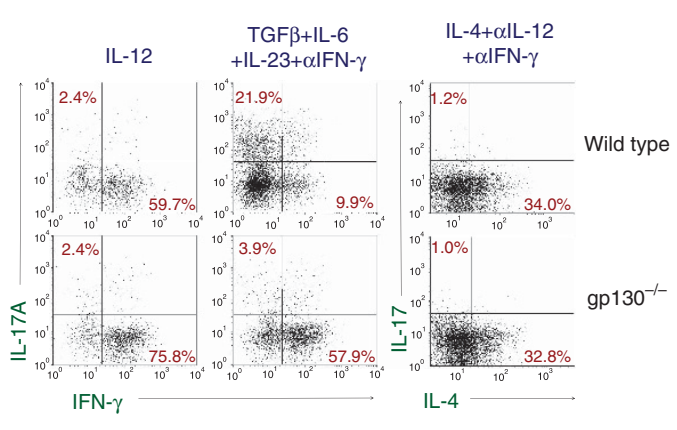

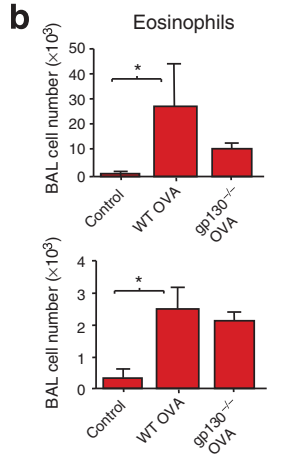
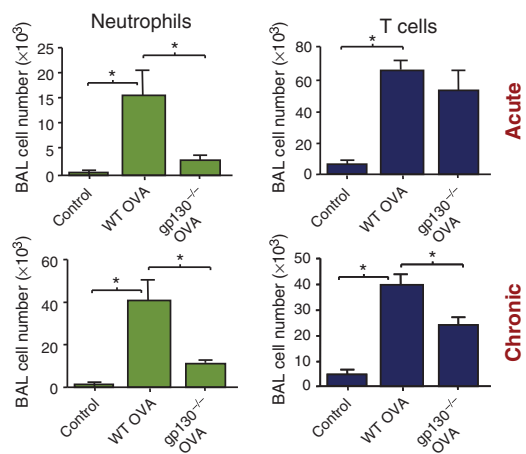
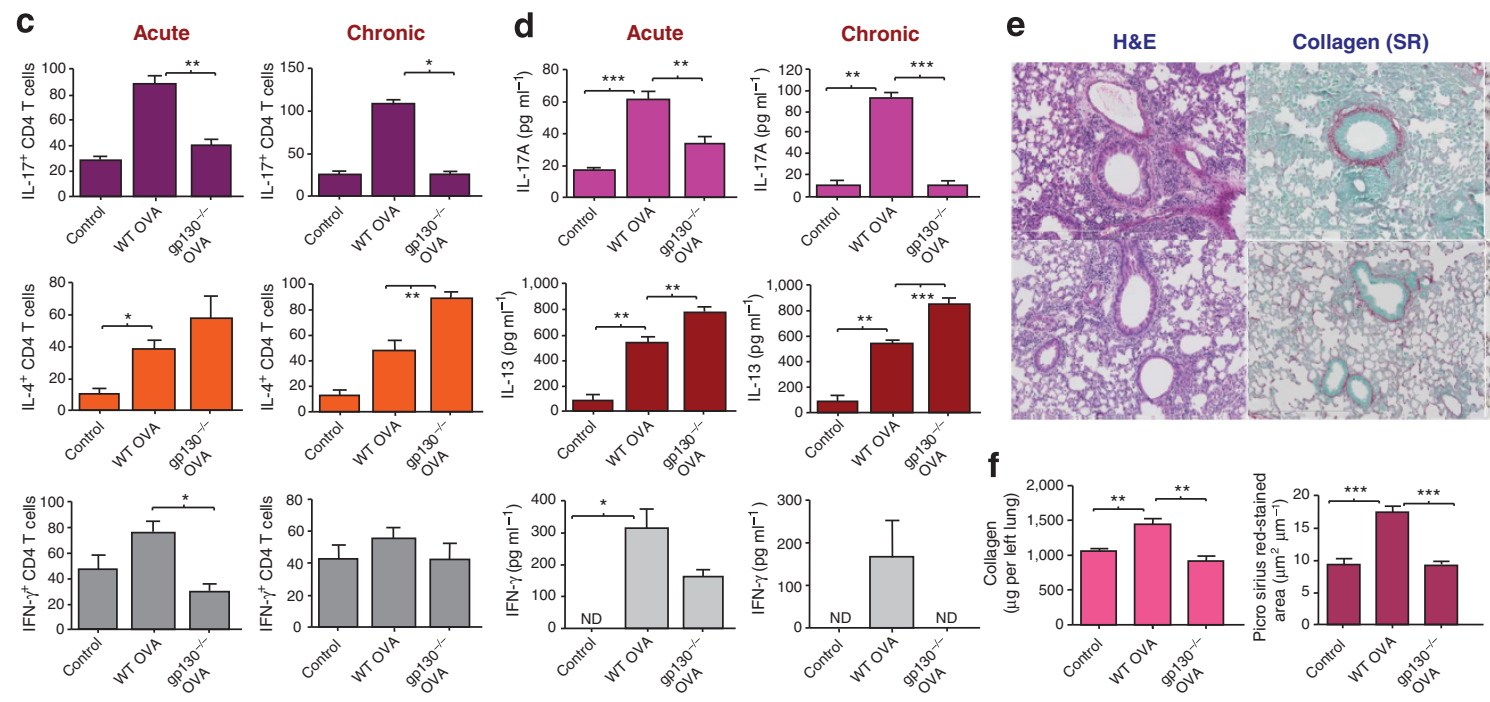

$\alpha-S M A$
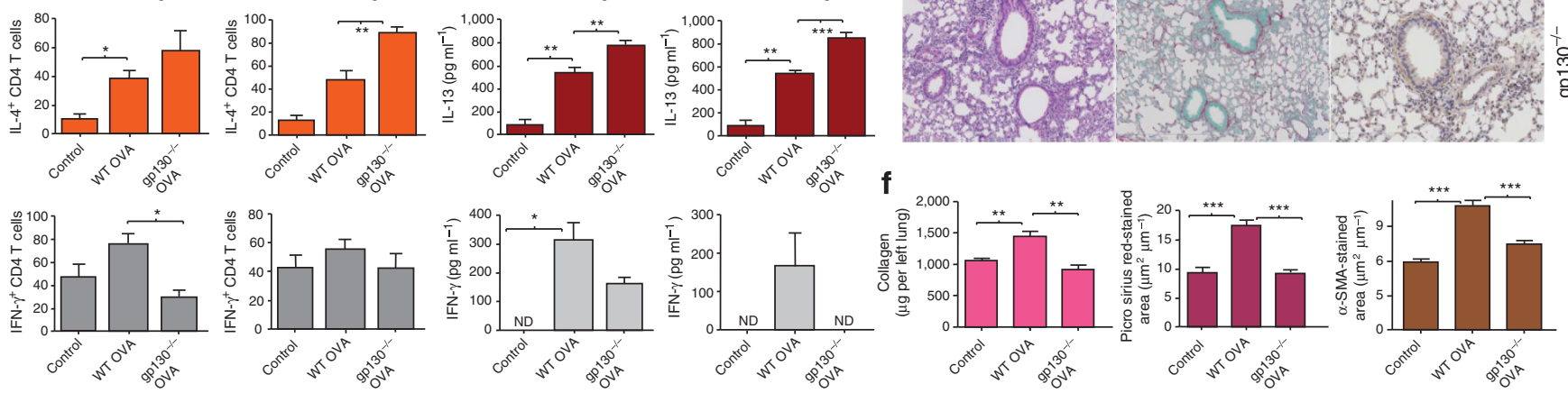

Figure 5 T-helper (Th)17 deficiency caused by disrupted T-cell interleukin (IL)-6 signaling leads to reduced chronic airway pathology. (a) Cytokine staining in CD4 cells from gp130-/- (lower panels) or control (wild-type (WT)) mice cultured under Th1- (IL-12), Th17- (tumor growth factor- $\beta$ (TGF- $\beta$ ), IL-6/23, anti-interferon (IFN)- $\gamma$ ), or Th2- (IL-4, anti-IL-12/IFN- $\gamma$ ) polarizing conditions with anti-CD3+anti-CD28 for 5 days, representative of three experiments. (b-d) Altered airway inflammation in gp $130^{-/-}$mice. Groups of gp130-/- or WT mice were mucosally sensitized with ovalbumin (OVA)/ tumor necrosis factor (TNF)- $\alpha$ and challenged with OVA until day 13 (acute) or day 53 (chronic) time points ( $n=6$, representative of two experiments). (b) Infiltration of inflammatory cells in BAL; control animals received PBS only. (c) Numbers of Th17, Th2, and Th1 CD4 cells in BAL. (d) Cytokines in bronchoalveolar lavage (BAL) supernatants. (e and f) Th17 deficiency abrogates airway remodeling. Chronically challenged gp130-/- or WT groups as in $\mathbf{b}$ were assessed by (e) lung histology, with staining for collagen (sirius red) and $\alpha$-smooth muscle actin ( $\alpha$-SMA) quantified in $\mathbf{f}$. Panel $f$ also shows total lung collagen content. $N=6$, representative of two experiments. $\mathrm{H} \& \mathrm{E}$, hematoxylin and eosin. ${ }^{*} P<0.05 ;{ }^{\star \star} P<0.01 ;{ }^{* \star \star} P<0.005$.

The proportion of Foxp $3^{+}$CD4 Treg cells in LN was temporarily increased after a week of ATRA treatment (day 45 group; Figure 6e). Although there was no increase in the proportion of Foxp3 ${ }^{+}$Treg in lung tissue in any group, ATRA treatment did significantly increase the expression of GARP on lung CD4 cells, indicating increased Treg activation. Thus, ATRA treatment alters the balance of Th17 and Treg activity, but its beneficial effects may be partially abrogated by enhanced eosinophilia. Finally, airway remodeling appeared to be improved by ATRA treatment from day 26 (Figure 6f), although there was no significant change in quantified $\alpha$-smooth muscle actin (histological analysis is shown in Supplementary Figure S2 online).

\section{DEX enhances Th17 differentiation in vitro and fails to inhibit} neutrophilia in vivo despite reduced T-cell inflammation

As Th17 cells have been associated with glucocorticoid-resistant airway inflammation, ${ }^{11}$ we determined the effect of DEX on Th17 responses in vitro and in vivo. Addition of DEX to proliferating OT-2 CD4 cells in the presence of Th17-polarizing cytokines (TGF- $\beta$, IL-6, and IL-23) strongly enhanced Th17 differentiation (Figure 7a). Another drug with potent potentiating effects on Th17 development was UO126, an inhibitor of T-cell receptortriggered mitogen-activated protein kinase kinase/extracellular signal-regulated kinase signaling (Figure 7a). Th17 cells induced by these drugs also expressed IL-22 (Supplementary Figure $\mathbf{3} 3$ online). Addition of both drugs induced the highest levels of Th17 development and intracellular ROR $\gamma \mathrm{t}$ expression (Figure 7a, lower panels). These data indicated that glucocorticoid receptor and mitogen-activated protein kinase pathways are positive and negative regulators of Th17 development, respectively. When we examined the effect of in vivo DEX treatment on acute and chronic airway disease initiated by mucosal sensitization, we found that prolonged DEX treatment inhibited lung Th17 recruitment and chronic eosinophilia (Figure $\mathbf{7 b}$ and $\mathbf{c}$ ). However, neutrophilia was not 

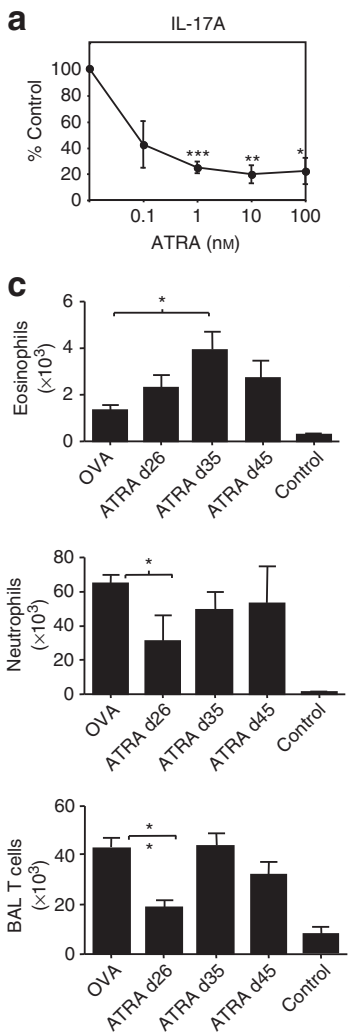

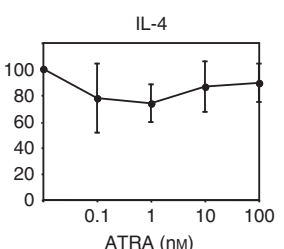

d
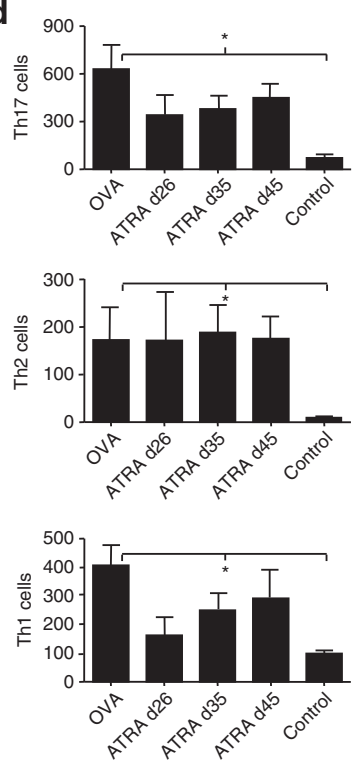

b
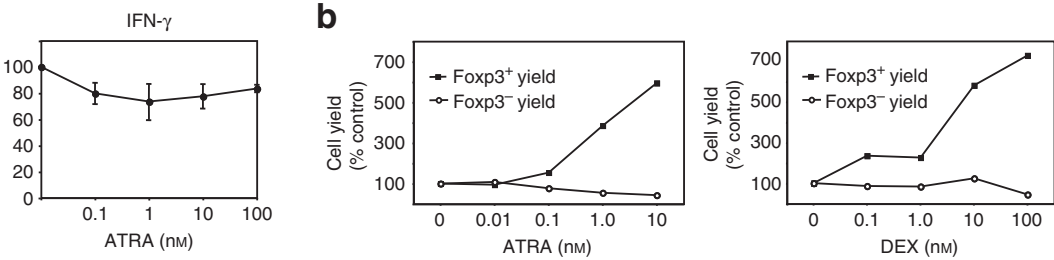

e
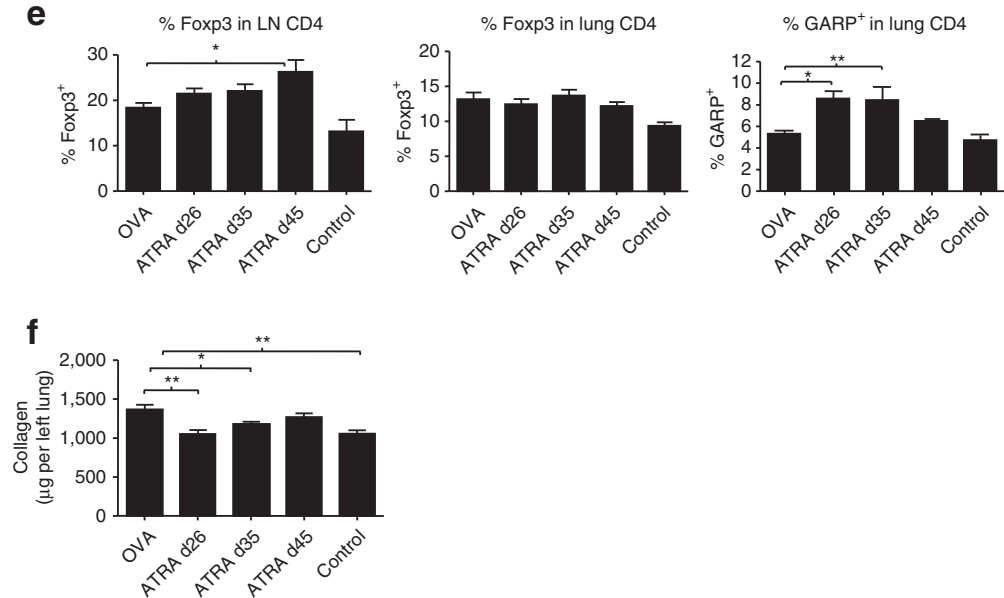

Figure 6 All-trans retinoic acid (ATRA) treatment boosts regulatory T-cell (Treg) activity and inhibits chronic T-helper (Th)17/neutrophil-associated airway remodeling. (a) Effect of ATRA on in vitro Th17, Th2, and Th1 development. OT-2 CD4 cells were cultured in polarizing conditions \pm different ATRA concentrations for 6 days before washing and restimulation for cytokine staining. Mean \pm s.e.m. from three experiments and expressed as \% control (no ATRA). (b) Effect of ATRA on CD4 Foxp3 ${ }^{+}$Treg expansion in vitro. CD25 ${ }^{+}$and CD25- CD4 cells were cultured with anti-CD3/ $28+$ interleukin (IL)-2, \pm ATRA, for 4 days, stained for Foxp3, and numbers of Foxp3 ${ }^{+}$and Foxp3 ${ }^{-}$cells in each culture counted by flow cytometry; representative of three experiments. (c-g) Effect of in vivo ATRA treatment, commenced on day 26 (d26), day 35 (d35), or day 45 (d45) of chronic ovalbumin (OVA)/tumor necrosis factor (TNF)- $\alpha$ protocol, on airway disease assessed on day 53. Panel c shows eosinophil, neutrophil, and T-cell infiltration, without (OVA) or with ATRA treatments. $N=5$, representative of two experiments. (d) Numbers of Th17, Th2, and Th1 cells in bronchoalveolar lavage (BAL). (e) Treg marker expression in CD4 populations from lung and lymph node (LN) cells. (f) Total lung collagen assay. Histological analysis of airway remodeling is shown in Supplementary Figure S2 online. DEX, dexamethasone; GARP, glycoprotein-A repetitions predominant; IFN, interferon. ${ }^{\star} P<0.05$; ${ }^{\star \star} P<0.01$; ${ }^{\star \star \star} P<0.005$.

inhibited by DEX. Short-term treatment of acute inflammation showed no significant inhibition by DEX. The increase in lung collagen content associated with airway remodeling was completely abrogated by DEX treatment (Figure 7d). Thus, certain aspects of Th17/neutrophil-associated disease, which increase with prolonged allergen exposure, may be refractory to inhibition by glucocorticoids. We therefore measured production of further cytokines IL-17F, IL-22, IL-25, and IL-33 in this model. These cytokines were undetectable in BAL fluid, but were secreted by draining LN cells after restimulation with OVA, with the exception of IL-17F, which was undetectable (Supplementary Figure $\mathbf{S 3}$ online). All cytokines were inhibited by in vivo DEX treatment. The distinct effect of DEX seen in vivo may be attributable to its ability to block expansion of OVA-specific $\mathrm{T}$ cells in LN, as shown in Supplementary Figure S3 online.

\section{DISCUSSION}

Asthma has traditionally been viewed as an allergic disease mediated by Th2 airway infiltration and eosinophilia, but a more complex set of pathologies is now apparent. Poor understanding of asthma subphenotypes and over-reliance on a simplified model of acute experimental allergic asthma might explain the poor translation of new asthma therapies to the clinic. While the presence of the Th17 cell in asthma has been postulated for some time, our study is the first to establish a direct role for this pathway in airway remodeling and to examine immunomodulatory strategies for targeting Th17 cells in a chronic disease model. Th17 cells and IL-17 can play beneficial as well as detrimental roles in airway pathology. Predominance of the Th17 response in the lung may reflect that the Th17 phenotype is tailored to meet the needs of the respiratory mucosa, increasing immune surveillance while restricting tissue damage via curtailment of inflammation or cross-regulation of allergic responses. ${ }^{12,13}$ Nevertheless, it is clear from our data that chronic Th17 inflammation contributes to airway remodeling and persistence of allergen sensitivity with increasing neutrophilia. As IL-17 increases allergen transfer into lung tissue, allergendriven Th17 cells might also increase susceptibility of lung tissue 

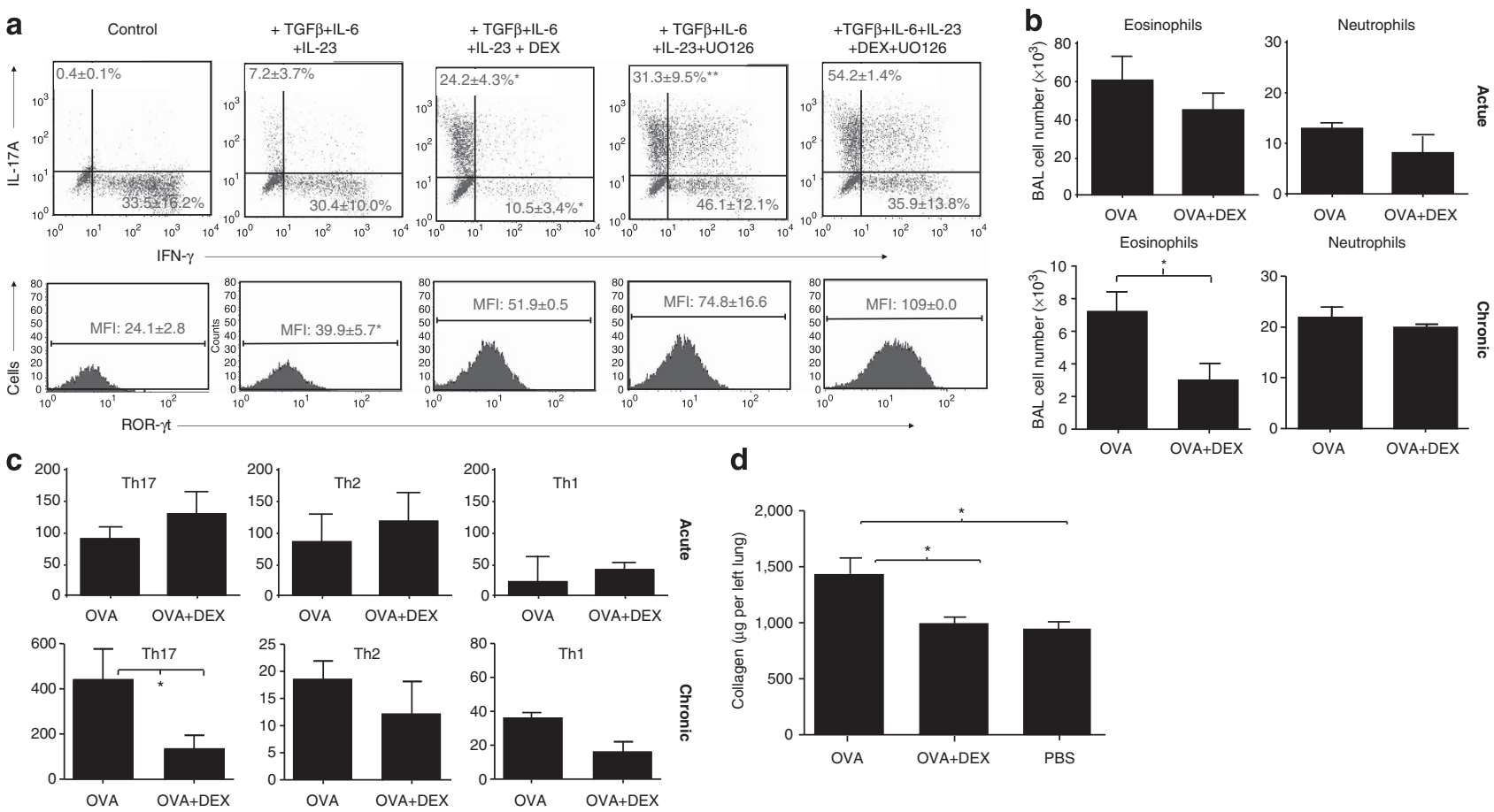

d
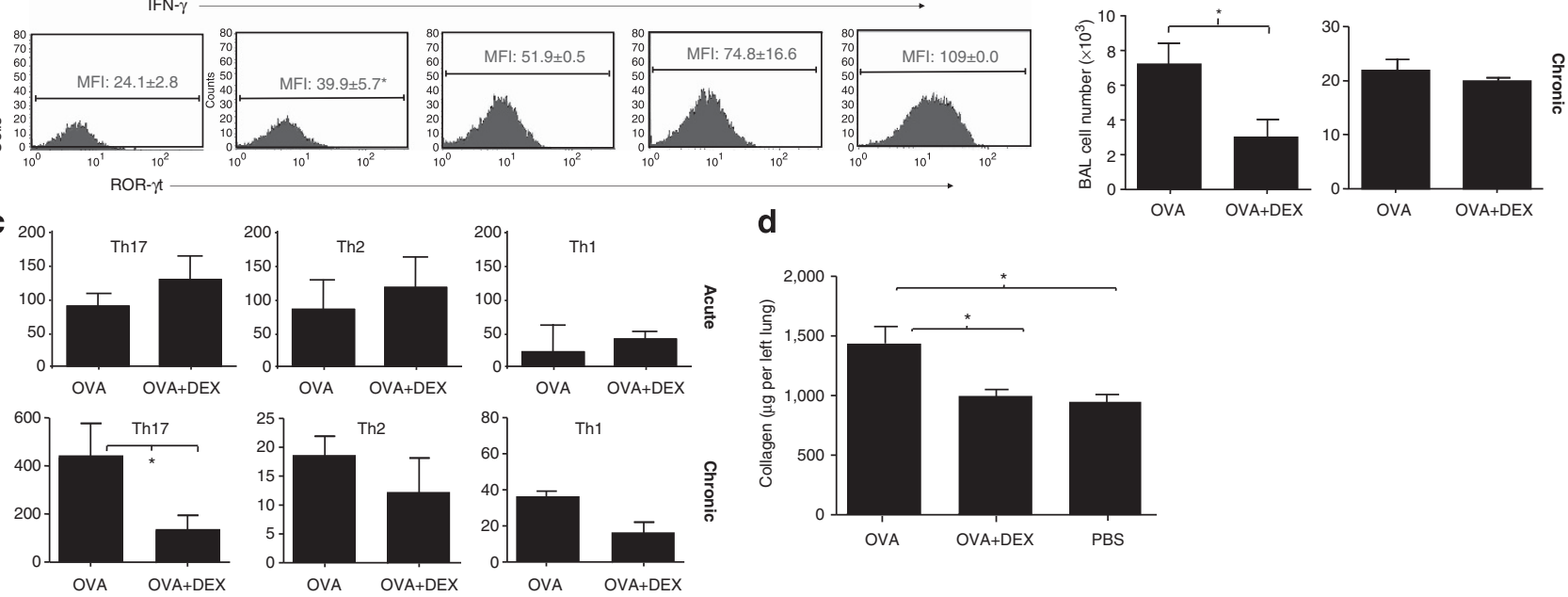

Figure 7 Regulation of T-helper (Th) 17 responses by glucocorticoid receptor and mitogen-activated protein kinase kinase (MEK) signaling pathways. (a) In vitro Th17 differentiation from OT-2 CD4 cells is enhanced by dexamethasone (DEX, 10 nM) and MEK inhibitor (UO126, $3 \mu \mathrm{g} \mathrm{ml}{ }^{-1}$ )- upper panel shows intracellular cytokine staining and lower panels show intracellular ROR $\gamma$ t staining, means \pm s.e.m. from five (upper panels) or three (lower panels) experiments. Statistical comparisons are to transforming growth factor (TGF)- $/$ /interleukin (IL)-6/IL-23-treated (upper) or control (lower). (b) Effect of in vivo DEX treatment on acute (upper graphs) and chronic (lower graphs) airway inflammation induced by ovalbumin (OVA)+tumor necrosis factor (TNF)- $\alpha$ mucosal sensitization, followed by OVA $(50 \mu \mathrm{g}) \pm \mathrm{DEX}(2 \mu \mathrm{g})$ intranasal (i.n.) challenges. Numbers of bronchoalveolar lavage (BAL) cells are shown $(n=5)$. Similar data were obtained in two experiments. (c) Numbers of BAL Th1, Th2, and Th17 cells as in $\mathbf{b}$. Insufficient T cells were obtained from control mice for cytokine staining. (d) Effect of DEX treatment on collagen deposition induced by OVA +TNF- $\alpha$ mucosal sensitization and chronic OVA challenge. Control animals received phosphate-buffered saline (PBS) $(n=5)$. MFI, mean fluorescent intensity. ${ }^{*} P<0.05$.

to infection, resulting in asthma exacerbations ${ }^{22}$ or accelerated tissue damage.

In humans, immune responses to inhaled aeroallergens develop in the lung and draining LN. Many animal models bypass this route by using intraperitoneal injections of allergen in alum adjuvant. Some features of this model are inconsistent with human asthma, e.g., airway eosinophils can reach $80 \%,{ }^{23}$ whereas in patients it is rarely $>5 \% .{ }^{24}$ The mixed Th17/Th2 response we observed was apparent even in primary T-cell responses in draining $\mathrm{LN}$ without adjuvant. Tolerance to inhaled OVA in naïve mice did induce T-cell activity and influx into the lung, but this involved Treg and not $\mathrm{T}$ effectors. Foxp $3^{+}$Treg appear to be generated in respiratory $\mathrm{LN}$ in response to allergen, recruited to the lung and then further activated by allergen. We demonstrated priming for IL-10 and IFN- $\gamma$ production during tolerance, both cytokines known to block allergic inflammation and/or Th17 responses. ${ }^{25-27}$ We could not determine whether the allergen-induced Treg were derived from the natural or peripherally induced Foxp $3^{+}$population. However, as they expressed high Helios levels during tolerance and thymic Treg from OT-2 transgenic mice proliferated extensively upon exposure to inhaled OVA, it seems most likely they are derived from natural Treg.
Our novel observations on Treg activity and phenotype in inflamed lungs compared with tolerance may help in the development of immunotherapeutic strategies. The inflammatory milieu of the lung appeared to block the ability of Treg to respond to allergen and this state of "paralysis" was associated with reduced Helios expression, a molecule recently associated with suppressive activity. ${ }^{28}$ Although it is possible that the lack of response to OVA was due to a lack of OVA-specificity (i.e., inflamed lungs recruited large numbers of nonspecific Treg), OVA-responsive Treg continued to be generated in LN. Perhaps, a short-lived inflammatory mediator present in lung tissue, but not reaching LN, was inhibiting local Treg activity. Reversing this hypothetical state of paralysis in local tissue Treg could potentially assist resolution of established disease.

The OVA/TNF mucosal sensitization model of chronic allergen exposure employed here resulted in less airway remodeling than OVA/alum, but may be closer to the human situation and mimic the prominent neutrophilic response seen in some severe asthmatics. ${ }^{29}$ This model recapitulates many clinical features of severe asthma, including closely associated IL-17 and neutrophil levels, ${ }^{30}$ increasing Th17 markers with increasing severity of disease, increased expression of IL-23 and $\mathrm{ROR} \gamma \mathrm{t},{ }^{31}$ and early airway remodeling. ${ }^{32}$ It therefore provides 
a potentially useful model for testing new asthma treatments, which can be introduced after initiation of remodeling. Use of OVA rather than house dust mite, which has natural adjuvanticity, allowed us to compare directly tolerance with inflammation in response to the same allergen, revealing that IL-17, like TNF- $\alpha$, can abrogate tolerance. Activation of epithelium by IL-17 and increased epithelial permeability could expose lung tissue to more allergen-associated or other danger signals in the environment, blocking Treg activity. IL-17 also abrogated priming of CD 4 cells for IL-10 and IFN- $\gamma$, associated with tolerance. Thus, as well as reciprocal expression of ROR $\gamma \mathrm{t}$ and Foxp 3 in developing allergen-specific T cells, cross-regulation between IL-17 and IL-10 may underlie the inability of sensitized mice to resolve inflammation during persistent allergen exposure. This provides a rationale for therapeutic blockade of both IL-6 and IL-17.

IL-17 has a putative role in acute severe and chronic asthma. ${ }^{33,34}$ B6 mice demonstrate airway hyper-reactivity during acute disease, but this is much reduced in the chronic phase. We therefore did not study airway hyper-reactivity in chronic disease, but focused on remodeling. We showed that IL-17 is increased alongside remodeling, and that allergen-driven Th17 cells rather than other sources of IL-17 are capable of inducing remodeling. Although some murine dendritic cells express CD4 and could potentially be affected by the mutation in gp $130^{-/-}$ mice, the profound defect we demonstrated in Th17 development is a more likely explanation for the lack of remodeling observed in this strain. Whether Th17 cells induce structural changes directly or via recruitment of neutrophils is not clear as there was a strong correlation between Th17 cells and neutrophilia.

Despite coexistence of Th17 and Th2 cells in inflamed tissues, there is some cross-regulation between Th17 and other subsets, explaining beneficial effects of Th17 development on Th1/Th2-mediated disease. However, models where plasticity between Th17 and Th1/Th2 cells are prominent provide evidence for pathogenicity of Th17s. The late appearance of a Th1 response in the lung after chronic allergen challenge seen here (Figure 2) has been observed previously. ${ }^{35}$ It may reflect deviation from a Th17 to Th1 phenotype or may be a consequence of remodeling and epithelial dysfunction, leading to a breakdown of mechanisms blocking Th1 responses in the lung. Interestingly, it has been proposed that only fully mature Th17, after exposure to IL-23, produce Th1 cytokine TNF- $\alpha$, and IL-22. ${ }^{36}$ Since neither cytokine was detected in lung Th17 cells but both were produced by in vitro-generated cells, in vitro derived Th17 used for adoptive transfer studies ${ }^{11}$ may be more differentiated and pathogenic than natural lung Th17. Clearly, TNF- $\alpha$ production would have a potent sensitizing effect on the airway. Nevertheless, IL-17 alone was sufficient to abrogate respiratory tolerance, probably via activation of epithelial cells that strongly express IL-17 receptor. ${ }^{37}$

Th17-deficient animals were skewed towards Th2 immunity compared with WT. Therefore, a strong Th17 response to allergen may be beneficial in acute disease due to inhibition of more damaging Th2-mediated pathology, as proposed. ${ }^{13}$
However, failure to resolve acute Th17 inflammation can clearly lead to long-term tissue damage and remodeling. As remodeling is such a prominent feature of human asthma, our data support the idea that Th17 pathways could be therapeutic targets in asthma. Natural Treg expansion was enhanced by ATRA, and in vivo ATRA treatment increases proportions of Treg in LN while enhancing their activation (reflected by GARP expression) in the lung. GARP (LRRC32) is an essential receptor for latent TGF- $\beta$ on the surface of active Treg ${ }^{19}$ and is related to suppressive activity. ${ }^{38}$ Treating mice with ATRA during the allergen challenge period ameliorated chronic disease despite exacerbating eosinophilia. This was not associated with an increase in Th2 cells and may have been due to ATRA directly enhancing eosinophil survival. ${ }^{39}$ Our data indicate that IL-17 blockade could ameliorate or exacerbate disease dependent on the type of patient, and like retinoic acids, should be combined with a therapy that blocks Th2 inflammation.

Although DEX enhanced Th17 development in vitro, prolonged treatment with the drug in vivo inhibited Th17-mediated inflammation in chronic disease. This was probably due to the multiple effects of glucocorticoid on diverse cell types including dendritic cells, which could have restricted expansion of allergen-specific T cells and their recruitment to the lung. However, the in vitro experiments, in which excess allergen was provided to the cultures, would suggest that activity of established Th17 effectors could be resistant to glucocorticoid. Chronic neutrophilia was uninhibited by DEX treatment, in contrast to eosinophilia. This could be due to resistance of Th17 cell activity to DEX inhibition or resistance of neutrophils to DEX-mediated apoptosis. ${ }^{40}$ Although our data do not support the idea that glucocorticoid treatment could exacerbate Th17/ neutrophilic components of asthma, they do suggest that other therapies may be indicated for treatment of severe/neutrophilic asthmatics. Most severe, treatment refractory asthma patients have mixed neutrophilia/eosinophilia, while around $40 \%$ have only neutrophilia. ${ }^{41}$ The latter group may benefit most from Th17-targeted therapies.

\section{METHODS}

Mice. All experiments were approved by our Institutional Animal Welfare Committee under UK Home Office Regulations. C57BL/6 (B6) mice (Harlan, Blackthorn, UK) were used at $4-8$ weeks. OT-2 T-cell receptor transgenic mice, specific for $\mathrm{OVA}^{323-339} / \mathrm{I}-\mathrm{A}^{\mathrm{b}}$ and CD4-Cre ${ }^{+/-}$ Xgp130 flox/flox mice, provided by Dr Werner Müller (University of Manchester, Manchester, UK) were on a B6 background. Homozygous CD4-Cre ${ }^{+/+}$Xgp130 flox/flox mice, with a deletion of the gp130 IL-6 receptor signaling component (IL-6 signal transducer) specifically in T cells, were used as a Th17-deficient $\operatorname{strain}^{20}$ and designated gp $130^{-1-}$. Control groups in these experiments were CD4-Cre ${ }^{-/-} \mathrm{Xgp} 130^{\text {flox/flox }}$ and designated WT.

Assessment of primary allergen responses. MACS-purified CD4 T cells from OT-2 LN + spleen cells were labeled with $5 \mu \mathrm{M}$ CFSE and transferred into the tail vein of B6 recipients given $50 \mu \mathrm{g} \mathrm{OVA} \pm 1 \mu \mathrm{g}$ rmTNF- $\alpha$ in $50 \mu$ PBS intranasally. After 3 days, mediastinal LN cells were stimulated with phorbol 12-myristate 13 -acetate (PMA) $\left(10 \mathrm{ng} \mathrm{ml}^{-1}\right)+$ ionomycin $\left(400 \mathrm{ng} \mathrm{ml}^{-1}\right)+$ monensin $(3 \mu \mathrm{M})$ for $5 \mathrm{~h}$ before intracellular cytokine staining. 
Induction of acute and chronic allergic airway inflammation. For parenteral sensitization protocols, mice were sensitized intraperitoneally with $10 \mu \mathrm{g}$ OVA in $0.2 \mathrm{ml}$ alum or complete Freund's adjuvant or intradermally in CASAC as described. ${ }^{42}$ Chronic challenge groups were immunized on days 0 and 12 . Controls received PBS. For mucosal sensitization, $10 \mu \mathrm{g}$ OVA $+1 \mu \mathrm{g}$ TNF- $\alpha$ was given intranasally on days 0 and 3 . Acute challenge mice received $50 \mu \mathrm{g}$ OVA intranasally on days $7,9-12$, and were killed on day 13. Chronic groups received $50 \mu \mathrm{g}$ OVA intranasally daily between days 17 and 21, thereafter three times a week from day 24 until day 53. All intranasal dosing was given in $50 \mu \mathrm{l}$ PBS per mouse under light-inhaled anesthesia (isoflurane). OVA (grade V; Sigma, Poole, UK) contained $0.4 \mu \mathrm{g}$ endotoxin per mg OVA protein.

Drug treatments. DEX (Sigma) was mixed with OVA and administered intranasally at $2 \mu$ g per mouse. ATRA (Sigma) in dimethylsulfoxide/olive oil was given intraperitoneally at $100 \mu \mathrm{g}$ per mouse, and controls received carrier alone. rmIL- 17 was given at $0.5 \mu \mathrm{g}$ IL- $17 \pm 50 \mu \mathrm{g}$ Alexa 488 -labeled OVA intranasally.

Histopathology and immunostaining of lungs. Paraffin sections were stained with hematoxylin and eosin or Picro-sirius red. For $\alpha$-smooth muscle actin staining, deparaffinized sections were incubated with anti$\alpha$-smooth muscle actin (1/250; Abcam, Cambridge, UK) or control rabbit Ig revealed with VECTASTAIN ABC kit (Vector Labs, Peterborough, UK). Peribronchiolar collagen deposition and smooth muscle were quantitated using the ImageScope image analysis software (Aperio Technologies, Vista, CA), with virtual slides generated by a NanoZoomer 2.0-HT scanner (Hamamatsu Photonics, Welwyn Garden City, UK). Circular lines were drawn around the outer and inner bronchiole of five bronchioles with a 150-200 $\mu \mathrm{m}$ diameter at comparable sites for each slide, using $\times 400$ original magnification. The area of specific staining within the airway wall was calculated and divided by the length of the basement membrane for each bronchiole $\left(\mu \mathrm{m}^{2}\right.$ per $\left.\mu \mathrm{m}\right)$.

Collagen assay. Total lung collagen content was measured by Sircol kit (Biocolor, Carrickfergus, UK).

Flow cytometry. Antibody staining $(0.1 \mu \mathrm{g}$ per sample (eBioscience, Hatfield, UK)), except anti-IL-22 (Poly5164; BioLegend, London, UK), anti-IL-4 (BVD6-24G2; Caltag, Buckingham, UK), and anti-CCR6 (29-2L17; BioLegend) was analyzed on a FACScalibur (BD, Oxford, UK). Inflammatory cells in BAL were identified as in ref.43 with the addition of anti-Gr- 1 to identify neutrophils $\left(\mathrm{Gr}-1^{+} \mathrm{CD} 11 \mathrm{c}^{-} \mathrm{CCR} 3^{-} \mathrm{CD} 4^{-} \mathrm{CD} 8^{-}\right.$ $\left.\mathrm{B} 220^{-}\right)$. BAL T cells were separated with anti-CD3/28 Dynabeads and cultured with $3 \mu \mathrm{m}$ monensin for $5 \mathrm{~h}$. Total cell numbers were calculated by analysis of fixed sample volumes, validated with fluorescent beads. Lung T cells were stimulated with immobilized anti-CD3 $\left(1 \mu \mathrm{g} \mathrm{ml}{ }^{-1}\right)+$ antiCD28 $\left(1 \mu \mathrm{g} \mathrm{ml}^{-1}\right)+$ monensin $(3 \mu \mathrm{M})$ or OVA $\left(500 \mu \mathrm{g} \mathrm{ml}^{-1}\right)+$ anti-CD28/ monensin for $12 \mathrm{~h}$ before intracellular cytokine staining as described. ${ }^{44}$ Intracellular staining protocol for Foxp 3 and Helios in CD25 ${ }^{+}$Treg was as described. ${ }^{44}$

BAL and LN supernatant enzyme-linked immunosorbent assay. Enzyme-linked immunosorbent assays were performed on BAL fluid or supernatants from mediastinal LN cells cultured $\left(2 \times 10^{6} \mathrm{ml}^{-1}\right)$ for $72 \mathrm{~h}$ with OVA $\left(500 \mu \mathrm{g} \mathrm{ml}^{-1}\right)$, as described, ${ }^{45}$ using antibody pairs for IL-17A (R\&D, Abingdon, UK), IL-23 (eBioscience), IFN- $\gamma$ (BD), IL-13 (Arcus, Modena, Italy), and eBioscience kits for IL-17F, IL-22, IL-25, and IL-33.

In vitro differentiation. Th17 cells were generated from naïve OT-2 $\mathrm{CD} 4^{+} \mathrm{CD} 44^{\text {lo }} \mathrm{T}$ cells + splenic APC in XVIVO-15+ OVA $323-339$ $\left(2 \mu \mathrm{g} \mathrm{ml}^{-1}\right)+\operatorname{hTGF} \beta\left(3 \mathrm{ng} \mathrm{ml}^{-1}\right)+\mathrm{IL}-1 \beta\left(10 \mathrm{ng} \mathrm{ml}^{2}, \mathrm{IL}-6\left(20 \mathrm{ng} \mathrm{ml}^{-1}\right)+\right.$ IL-23 $\left(10 \mathrm{ng} \mathrm{ml}^{-1}\right) \pm \mathrm{DEX}(10 \mathrm{nM})$, or UO126 $\left(3 \mu \mathrm{g} \mathrm{ml}^{-1}\right.$; Calbiochem, Nottingham, UK). Th1 cultures contained IL-12 $\left(10 \mathrm{ng} \mathrm{ml}^{-1}\right)$ and Th2 IL-4 $\left(10 \mathrm{ng} \mathrm{ml}^{-1}\right)+$ anti-IL-12/IFN- $\gamma\left(2.5 \mu \mathrm{g} \mathrm{ml}^{-1}\right)$.
Statistical analysis. Data are means \pm s.e.m. Significance between groups was assessed using Student's $t$-tests, except where s.d.'s were significantly different, in which case Mann-Whitney tests were used. ${ }^{\star} P<0.05$, ${ }^{* *} P<0.01$ and ${ }^{* * *} P<0.005$ in all figures.

SUPPLEMENTARY MATERIAL is linked to the online version of the paper at http://www.nature.com/mi

\section{ACKNOWLEDGMENTS}

This work was funded by Asthma UK grant 07/022. We thank W. Muller

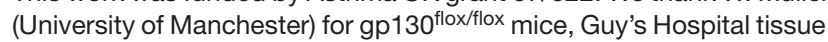
bank team for help with histology, and S. Saglani and S. Ying for advice on analysis of airway remodeling.

\section{DISCLOSURE}

The authors declared no conflict of interest.

(C) 2013 Society for Mucosal Immunology

\section{REFERENCES}

1. Kumar, R.K., Herbert, C., Webb, D.C., Li, L. \& Foster, P.S. Effects of anticytokine therapy in a mouse model of chronic asthma. Am. J. Respir. Crit. Care Med. 170, 1043-1048 (2004).

2. Humbles, A.A. et al. A critical role for eosinophils in allergic airways remodeling. Science 305, 1776-1779 (2004).

3. McMillan, S.J., Xanthou, G. \& Lloyd, C.M. Manipulation of allergeninduced airway remodeling by treatment with anti-TGF- $\beta$ antibody: effect on the Smad signaling pathway. J. Immunol. 174, 5774-5780 (2005).

4. Saglani, S., Mathie, S.A., Gregory, L.G., Bell, M.J., Bush, A. \& Lloyd, C.M. Pathophysiological features of asthma develop in parallel in house dust mite exposed neonatal mice. Am. J. Respir. Cell Mol. Biol. 41, 281-289 (2009).

5. Schnyder-Candrian, S. et al. Interleukin-17 is a negative regulator of established allergic asthma. J. Exp. Med. 203, 2715-2725 (2006).

6. Doe, C. et al. Expression of the Thelper 17-associated cytokines IL-17A and IL-17F in asthma and COPD. Chest 138, 1140-1147 (2010).

7. Nakae, S. et al. Antigen-specific T cell sensitization is impaired in IL-17 deficient mice, causing suppression of allergic cellular and humoral responses. Immunity 17, 375-387 (2002).

8. Fossiez, F. et al. T cell interleukin-17 induces stromal cells to produce proinflammatory and hematopoietic cytokines. J. Exp. Med. 183, 2593-2603 (1996).

9. Vanaudenaerde, B.M., Wuyts, W.A., Dupont, L.J., Van Raemdonck, D.E., Demedts, M.M. \& Verleden, G.M. Interleukin-17 stimulates release of interleukin-8 by human airway smooth muscle cells in vitro: a potential role for interleukin-17 and airway smooth muscle cells in bronchiolitis obliterans syndrome. J. Heart Lung Transplant 22, 1280-1283 (2003).

10. Molet, S. et al. IL-17 is increased in asthmatic airways and induces human bronchial fibroblasts to produce cytokines. J. Allergy Clin. Immunol. 108, 430-438 (2001).

11. McKinley, L. et al. Th17 cells mediate steroid-resistant airway inflammation and airway hyperresponsiveness in mice. J. Immunol. 181, 4089-4097 (2008).

12. Murdoch, J.R. \& Lloyd, C.M. Resolution of allergic airway inflammation and airway hyperreactivity is mediated by $\mathrm{IL}-17$-producing $\gamma \delta \mathrm{T}$ cells. Am. J. Respir. Crit. Care Med. 182, 464-476 (2010).

13. Barlow, J.L., Flynn, R.J., Ballantyne, S.J. \& McKenzie, A.N. Reciprocal expression of IL-25 and IL-17A is important for allergic airways hyperreactivity. Clin. Exp. Allergy 41, 1447-1455 (2011).

14. Thornton, A.M. et al. Expression of Helios, an Ikaros transcription factor family member, differentiates thymic-derived from peripherally induced Foxp3+ T regulatory cells. J. Immunol. 184, 3433-3441 (2010).

15. Yokota, S. et al. Efficacy and safety of tocilizumab in patients with systemic-onset juvenile idiopathic arthritis: a randomised, double-blind, placebo-controlled, withdrawal phase III trial. Lancet 371, 998-1006 (2008).

16. Piggott, D.A. et al. MyD88-dependent induction of allergic Th2 responses to intranasal antigen. J. Clin. Invest 115, 459-467 (2005). 
17. Reboldi, A. et al. C-C chemokine receptor 6-regulated entry of Th17 cells into the CNS through the choroid plexus is required for the initiation of EAE. Nat. Immunol. 10, 514-523 (2009).

18. Wells, J.W., Cowled, C.J., Farzaneh, F. \& Noble, A. Combined triggering of dendritic cell receptors results in synergistic activation and potent cytotoxic immunity. J. Immunol. 181, 3422-3431 (2008).

19. Tran, D.Q., Andersson, J., Wang, R., Ramsey, H., Unutmaz, D. \& Shevach, E.M. GARP (LRRC32) is essential for the surface expression of latent TGF- $\beta$ on platelets and activated FOXP3 ${ }^{+}$regulatory $T$ cells. Proc. Natl. Acad. Sci. USA 106, 13445-13450 (2009).

20. Fasnacht, N., Greweling, M.C., Bollati-Fogolin, M., Schippers, A. \& Muller W. T-cell-specific deletion of gp130 renders the highly susceptible IL-10deficient mouse resistant to intestinal nematode infection. Eur. J. Immunol. 39, 2173-2183 (2009)

21. Mucida, D. et al. Reciprocal Th17 and regulatory T cell differentiation mediated by retinoic acid. Science 317, 256-260 (2007).

22. Bartlett, N.W. et al. Mouse models of rhinovirus-induced disease and exacerbation of allergic airway inflammation. Nat. Med. 14, 199-204 (2008)

23. Kung, T.T. et al. Characterization of a murine model of allergic pulmonary inflammation. Int. Arch. Allergy Immunol. 105, 83-90 (1994).

24. Lex, C. et al. Airway eosinophilia in children with severe asthma: predictive values of noninvasive tests. Am. J. Respir. Crit. Care Med. 174, 1286-1291 (2006)

25. Akbari, O. et al. Antigen-specific regulatory T cells develop via the ICOSICOS-ligand pathway and inhibit allergen-induced airway hyperreactivity. Nat. Med. 8, 1024-1032 (2002).

26. Yoshida, M. et al. Effect of interferon- $\gamma$ on allergic airway responses in interferon- $\gamma$-deficient mice. Am. J. Respir. Crit. Care Med. 166, 451-456 (2002).

27. Harrington, L.E. et al. Interleukin 17-producing CD4 ${ }^{+}$effector T cells develop via a lineage distinct from the Thelper type 1 and 2 lineages. Nat. Immunol. 6, 1123-1132 (2005)

28. Zabransky, D.J. et al. Phenotypic and functional properties of Helios ${ }^{+}$ regulatory T cells. PLoS One 7, e34547 (2012)

29. Holgate, S.T. \& Polosa, R. The mechanisms, diagnosis, and management of severe asthma in adults. Lancet 368, 780-793 (2006).

30. Bullens, D.M. et al. IL-17 mRNA in sputum of asthmatic patients: linking T cell driven inflammation and granulocytic influx? Respir. Res. 7, 135 (2006).

31. Zhao, Y., Yang, J., Gao, Y.D. \& Guo, W. Th17 immunity in patients with allergic asthma. Int. Arch. Allergy Immunol. 151, 297-307 (2010).
32. Saglani, S. et al. Early detection of airway wall remodeling and eosinophilic inflammation in preschool wheezers. Am. J. Respir. Crit. Care Med. 176, 858-864 (2007).

33. Linden, A. Role of interleukin-17 and the neutrophil in asthma. Int. Arch. Allergy Immunol. 126, 179-184 (2001).

34. Al-Ramli, W. et al. Th17-associated cytokines (IL-17A and IL-17F) in severe asthma. J. Allergy Clin. Immunol. 123, 1185-1187 (2009).

35. McMillan, S.J. \& Lloyd, C.M. Prolonged allergen challenge in mice leads to persistent airway remodelling. Clin. Exp. Allergy 34, 497-507 (2004).

36. Korn, T., Bettelli, E., Oukka, M. \& Kuchroo, V.K. IL-17 and Th17 cells. Annu. Rev. Immunol. 27, 485-517 (2009).

37. McAllister, F. et al. Role of IL-17A, IL-17F, and the IL-17 receptor in regulating growth-related oncogene-alpha and granulocyte colonystimulating factor in bronchial epithelium: implications for airway inflammation in cystic fibrosis. J. Immunol. 175, 404-412 (2005)

38. Chan, D.V. et al. Signal peptide cleavage is essential for surface expression of a regulatory $T$ cell surface protein, leucine rich repeat containing 32 (LRRC32). BMC Biochem. 12, 27 (2011).

39. Ueki, S. et al. Retinoic acids are potent inhibitors of spontaneous human eosinophil apoptosis. J. Immunol. 181, 7689-7698 (2008).

40. Meagher, L.C., Cousin, J.M., Seckl, J.R. \& Haslett, C. Opposing effects of glucocorticoids on the rate of apoptosis in neutrophilic and eosinophilic granulocytes. J. Immunol. 156, 4422-4428 (1996).

41. Wenzel, S.E. et al. Evidence that severe asthma can be divided pathologically into two inflammatory subtypes with distinct physiologic and clinical characteristics. Am. J. Respir. Crit. Care Med. 160, 1001-1008 (1999)

42. Wells, J.W., Choy, K., Lloyd, C.M. \& Noble, A. Suppression of allergic airway inflammation and lgE responses by a class I restricted allergen peptide vaccine. Mucosal Immunol. 2, 54-62 (2009).

43. van Rijt, L.S., Kuipers, H., Vos, N., Hijdra, D., Hoogsteden, H.C. \& Lambrecht, B.N. A rapid flow cytometric method for determining the cellular composition of bronchoalveolar lavage fluid cells in mouse models of asthma. J. Immunol. Methods 288, 111-121 (2004)

44. Giorgini, A. \& Noble, A. Blockade of chronic graft-versus-host disease by alloantigen-induced $\mathrm{CD} 4^{+} \mathrm{CD} 25^{+}$Foxp3 ${ }^{+}$regulatory $\mathrm{T}$ cells in non-lymphopenic hosts. J. Leukoc. Biol. 82, 1053-1061 (2007)

45. Thomas, M.J., Noble, A., Sawicka, E., Askenase, P.W. \& Kemeny, D.M CD8 T cells inhibit lgE via dendritic cell IL-12 induction that promotes Th T cell counter-regulation. J. Immunol. 168, 216-223 (2002). 